University of Nebraska - Lincoln

DigitalCommons@University of Nebraska - Lincoln

Robert Streubel Papers

Research Papers in Physics and Astronomy

4-30-2012

\title{
Out-of-surface vortices in spherical shells
}

Volodymyr P. Kravchuk

Bogolyubov Institute for Theoretical Physics Nasu, vkravchuk@bitp.kiev.ua

Denis D. Sheka

Taras Shevchenko National University of Kyiv

Robert Streubel

Leibniz-Institut für Festkörper- und Werkstoffforschung Dresden, streubel@unl.edu

Denys Makarov

Leibniz-Institut für Festkörper- und Werkstoffforschung Dresden

Oliver G. Schmidt

Leibniz-Institut für Festkörper- und Werkstoffforschung Dresden

See next page for additional authors

Follow this and additional works at: https://digitalcommons.unl.edu/physicsstreubel

Part of the Atomic, Molecular and Optical Physics Commons, Condensed Matter Physics Commons, and the Other Physics Commons

Kravchuk, Volodymyr P.; Sheka, Denis D.; Streubel, Robert; Makarov, Denys; Schmidt, Oliver G.; and Gaididei, Yuri, "Out-of-surface vortices in spherical shells" (2012). Robert Streubel Papers. 24.

https://digitalcommons.unl.edu/physicsstreubel/24

This Article is brought to you for free and open access by the Research Papers in Physics and Astronomy at DigitalCommons@University of Nebraska - Lincoln. It has been accepted for inclusion in Robert Streubel Papers by an authorized administrator of DigitalCommons@University of Nebraska - Lincoln. 


\section{Authors}

Volodymyr P. Kravchuk, Denis D. Sheka, Robert Streubel, Denys Makarov, Oliver G. Schmidt, and Yuri Gaididei 


\title{
Out-of-surface vortices in spherical shells
}

\author{
Volodymyr P. Kravchuk, ${ }^{1, *}$ Denis D. Sheka, ${ }^{2}$ Robert Streubel, ${ }^{3,4}$ Denys Makarov, ${ }^{3}$ Oliver G. Schmidt, ${ }^{3,4}$ and Yuri Gaididei ${ }^{1}$ \\ ${ }^{1}$ Bogolyubov Institute for Theoretical Physics, 03143 Kiev, Ukraine \\ ${ }^{2}$ Taras Shevchenko National University of Kiev, 01601 Kiev, Ukraine \\ ${ }^{3}$ Institute for Integrative Nanosciences, IFW Dresden, 01069 Dresden, Germany \\ ${ }^{4}$ Material Systems for Nanoelectronics, Chemnitz University of Technology, 09107 Chemnitz, Germany
}

(Received 27 February 2012; published 30 April 2012)

\begin{abstract}
The interplay of topological defects with curvature is studied for out-of-surface magnetic vortices in thin spherical nanoshells. In the case of an easy-surface Heisenberg magnet it is shown that the curvature of the underlying surface leads to a coupling between the localized out-of-surface component of the vortex with its delocalized in-surface structure, i.e., polarity-chirality coupling.
\end{abstract}

DOI: 10.1103/PhysRevB.85.144433

PACS number(s): 75.10.Hk, 05.45.-a, 72.25.Ba, 75.40.Mg

\section{INTRODUCTION}

Understanding the interplay between geometry and topology of condensed matter order is of fundamental importance in several physical and biophysical contexts, and this combination raises a number of unsolved questions. Examples include thin layers of superfluids ${ }^{1}$ and superconductors, ${ }^{2,3}$ nematic liquid crystal shells, ${ }^{4}$ viral shells, ${ }^{5}$ and cell membranes. ${ }^{6} \mathrm{~A}$ considerable effort has been invested in understanding the role of the coupling between in-surface order and curvature of the underlying surface. ${ }^{7,8}$ The topological defects of $2 \mathrm{D}$ in-surface vector fields are characterized by a winding number for the phaselike variable: vorticity $q \in \mathbb{Z}$ [topological charge of $\pi_{1}\left(S^{1}\right)$ homotopy group]. On curved surfaces the Gaussian curvature leads to screening topological charges. ${ }^{9}$ Vortices in curved superfluid films are a typical example of such defects.

Vortices in magnets belong to a more general type of topological defect. In addition to the vorticity, the magnetic vortex is also characterized by the polarity $p= \pm 1$, which describes the vortex core magnetization. The topological properties of magnetic vortices are characterized by the relative homotopy group $\pi_{2}\left(S^{2}, S^{1}\right)^{10}$ and depend on both vorticity and polarity. Magnetic vortices were intensively studied during past few decades for the sake of applications in nanomagnetism as high-density magnetic storage devices ${ }^{11}$ and miniature sensors. ${ }^{12}$ Investigations of different aspects of magnetic vortex statics and dynamics were mainly restricted to flat structures. In such nanomagnets, the vortex appears as a ground state in sub-micrometer-sized magnets due to competition between short-range exchange interactions and long-range dipole interactions. ${ }^{12,13}$ The ground state of smaller samples is typically characterized by in-plane quasiuniform magnetization. Contrary to in-surface, a quasiuniform magnetization distribution in thin spherical shells is forbidden for topological reasons; instead, two oppositely disposed vortices are expected.

In a flat nanomagnet, the vortex state is degenerated with respect to polarity. Hence, one can link the vortex polarity to the bit of information with possible spintronics applications. ${ }^{14}$ One of the consequence of a more complicated topology of the magnetic vortex is a gyroscopical force that depends on both vorticity and polarity of the vortex. Therefore, the vortex polarity can be switched by exciting the gyroscopical motion.
The switching thresholds for the two polarities are equal only in ideally flat structures. ${ }^{15}$ Experiments on permalloy platelets have revealed a relatively large asymmetry in thresholds, ${ }^{15,16}$ which originates from the lack of the mirror symmetry of rough thin-film structures. ${ }^{17}$ This indicates the interplay between the vortex polarity and the curvature of the underlying surface.

The influence of a curvature on magnetic properties has been studied both experimentally and theoretically for geometries of cylinder, ${ }^{18}$ torus,${ }^{19}$ cone,${ }^{20}$ solid spheres, ${ }^{21}$ and hemispherical cap structures. ${ }^{22} \mathrm{~A}$ precise theoretical description of peculiarities of vortices on spherical surfaces is not available in literature. Most theoretical studies are limited to skyrmion-like solutions. $^{23}$

In this paper, we study the structure of magnetic vortices on a thin spherical shell with an easy-surface anisotropy. Using the anisotropic Heisenberg model, we find possible solutions of the vortex type. In contrast to vortices in flat magnets, there is an interplay between the localized out-of-surface and the delocalized in-surface structures. In other words, the vortex core plays the role of a charge source for the vortex phase structure.

\section{MODEL AND THE VORTEX SOLUTIONS}

The magnetic energy of a classical Heisenberg easy-surface ferromagnet has the following form:

$$
E=A \int d \boldsymbol{r}\left[-\boldsymbol{m} \cdot \nabla^{2} \boldsymbol{m}+\frac{(\boldsymbol{m} \cdot \boldsymbol{n})^{2}}{\ell^{2}}\right],
$$

with the exchange constant $A$, the anisotropy constant $K>0$, magnetic length $\ell=\sqrt{A / K}$, and the surface normal $\boldsymbol{n}$, and the integration is over the volume of the spherical shell. In the following, we use the local spherical reference frame for the unit magnetization vector $\boldsymbol{m}=\left(m_{r}, m_{\vartheta}, m_{\chi}\right)=$ $(\cos \Theta, \sin \Theta \cos \Phi, \sin \Theta \sin \Phi)$. Here the angular magnetic variables $\Theta=\Theta(\boldsymbol{r})$ and $\Phi=\Phi(\boldsymbol{r})$ describe the magnetization distribution with respect to the spherical coordinates $(r, \vartheta, \chi)$ of the radius-vector $\boldsymbol{r}$. Hereafter, we consider a case of thin and high anisotropy shells, $h \ll \ell \ll L$, where $h$ is thickness of the shell and $L$ is its inner radius. Therefore, we assume that the magnetization does not depend on the radial coordinate $r$. On the indicated conditions the total magnetic energy in terms 
of the local reference frame reads

$$
\begin{aligned}
E= & A h \int_{0}^{2 \pi} d \chi \int_{0}^{\pi} d \vartheta \sin \vartheta\left\{\left(\partial_{\vartheta} \Theta+\cos \Phi\right)^{2}\right. \\
& +\frac{1}{\sin ^{2} \vartheta}\left(\partial_{\chi} \Theta+\sin \vartheta \sin \Phi\right)^{2}+\frac{L^{2}}{\ell^{2}} \cos ^{2} \Theta \\
& +\sin ^{2} \Theta\left[\left(\partial_{\vartheta} \Phi-\sin \Phi \cot \Theta\right)^{2}\right. \\
& \left.\left.+\frac{1}{\sin ^{2} \vartheta}\left(\partial_{\chi} \Phi+\cos \vartheta+\sin \vartheta \cot \Theta \cos \Phi\right)^{2}\right]\right\} .
\end{aligned}
$$

In this case, the static magnetization configuration the energy functional (2) produces the Euler-Lagrange equations,

$$
\begin{gathered}
\nabla^{2} \Theta-\sin \Theta \cos \Theta\left[(\nabla \Phi)^{2}-1+\cot ^{2} \vartheta-\frac{L^{2}}{\ell^{2}}\right] \\
=2 \frac{\sin ^{2} \Theta}{\sin \vartheta}\left[\Xi \partial_{\chi} \Phi-\partial_{\vartheta}(\sin \vartheta \cos \Phi)\right], \\
\nabla \cdot\left(\sin ^{2} \Theta \nabla \Phi\right)=-2 \frac{\sin ^{2} \Theta}{\sin \vartheta}\left[\Xi \partial_{\chi} \Theta+\sin \vartheta \sin \Phi \partial_{\vartheta} \Theta\right],
\end{gathered}
$$

where $\Xi \equiv \cot \Theta \cot \vartheta-\cos \Phi$ and the $\nabla$ operators denotes the angular parts of the corresponding differential operators in the spherical local basis.

In the case of a high easy-surface anisotropy $(\ell \rightarrow 0)$, the solution of Eqs. (3) which minimize the energy (2) reads $\Theta=\pi / 2, \Phi=$ const. This is a vortex solution where the magnetization is confined within the sphere surface except for two diametrically opposite point singularities: vortex cores. Such "in-surface" vortices are well studied in different media. ${ }^{7,8}$ Here we demonstrate that taking into account the out-of-surface structure of the vortex core (finite $0<\ell \ll L$ ) essentially changes the vortex state properties in case of the curved surface as compared with planar magnets. ${ }^{24}$ In the following, we consider only the azimuthally symmetric vortex solution $\Theta=\Theta(\vartheta), \Phi=\Phi(\vartheta)$ by analogy with the planar vortices.

The out-of-surface magnetization of the vortex core, the so-called polarity, takes two values, $p= \pm 1$ (outward and inward). The magnetization distribution can be analyzed asymptotically near the vortex center $(\vartheta=0)$; see Appendix A. The size of the vortex core is determined by the vortex out-of-surface magnetization, $\cos \Theta \approx p\left(1-\vartheta^{2} / 2 \vartheta_{c}^{2}\right)$, with $\vartheta_{c} \ll 1$. Moreover, the in-surface magnetization is described by the angular variable $\Phi \approx \Phi_{0}-p \sin \Phi_{0} \vartheta^{2} /\left(4 \vartheta_{c}\right)$, where the constant $\Phi_{0}$ will be determined later. Although the asymptotic limit of out-of-surface component is similar to that of a vortex in planar magnets, ${ }^{25}$ the phase $\Phi$ depends on the vortex polarity $p$, which is a distinct feature compared with the constant value in planar vortices. Since the out-of-surface magnetization has an exponentially localized structure, the following ansatz function (similar to the vortices in planar magnets ${ }^{25}$ ) can be used for description:

$$
\cos \Theta=p_{1} e^{-\frac{1}{2}\left(\frac{\vartheta}{\vartheta_{c}}\right)^{2}}+p_{2} e^{-\frac{1}{2}\left(\frac{\pi-\vartheta}{\vartheta_{c}}\right)^{2}},
$$

where $p_{1}$ and $p_{2}$ are polarities of the vortices at the poles. Stability of the vortex solutions for the case $\ell \ll L$ was confirmed numerically using micromagnetic simulations; see the latter text. The obtained out-of-surface component of the vortices appears to be in good agreement with the ansatz (4); see Fig. 3. We now consider the vortex in-surface magnetization. Accurate within the vortex core corrections, the $\Phi$ distribution can be described by the following equation:

$$
\partial_{u u} \Phi=-g(u) \sin \Phi, \quad g(u)=-\frac{4 e^{u} \partial_{u} m_{r}(u)}{1+e^{2 u}},
$$

with $u=\ln \tan (\vartheta / 2)$ and $m_{r}(u)=\cos \Theta$. The function $g(u)$ consists of two peaks localized near the vortex cores at $u_{c} \approx$ $\ln \cot \left(\vartheta_{c} / 2\right)$.

In order to analyze the $\Phi$-distribution outside cores, we use the stepwise vortex shape model for the out-of-surface magnetization, $m_{r}(u) \approx p_{1}-p_{1} h\left(u+u_{c}\right)+p_{2} h\left(u-u_{c}\right)$ with the Heaviside step function $h(u)$. Using this approach, $g(u)$ becomes $g(u) \approx \frac{\pi}{2} \vartheta_{c}\left[p_{1} \delta\left(u+u_{c}\right)-p_{2} \delta\left(u-u_{c}\right)\right]$. The consequence of such a model is that the localized out-of-surface structure plays the role of the charge density for the delocalized in-surface structure. The solution of this model, which satisfies the Neumann boundary conditions $\partial_{u} \Phi( \pm \infty)=0$, has the implicit form,

$$
\begin{aligned}
\Phi(u)= & \Phi_{0}-\frac{\pi}{2} \vartheta_{c} p_{1} \sin \Phi\left(-u_{c}\right)\left[\left(u+u_{c}\right)_{+}-\left(u-u_{c}\right)_{+}\right] \\
& p_{1} \sin \Phi\left(-u_{c}\right)=p_{2} \sin \Phi\left(u_{c}\right)
\end{aligned}
$$

with $u_{+} \equiv u h(u)$. The further analysis essentially depends on the relative orientations of vortices.

For the case of same polarities $\left(p_{1}=p_{2}=p\right)$, the solution $\Phi$ that minimizes the energy (2) takes the following explicit form outside vortex cores $\left(\vartheta_{c} \ll 1\right)$ :

$$
\Phi(\vartheta) \approx \pm \frac{\pi}{2}\left(1-p \vartheta_{c} \alpha \ln \tan \frac{\vartheta}{2}\right),
$$

where $\alpha$ is solution of the equation $\alpha=\cos \left(\alpha \vartheta_{c} u_{c} \pi / 2\right)$; for details see Appendix B. Since $\vartheta_{c} u_{c} \ll 1$, then $\alpha \lesssim 1$. Accordingly, $\Phi$ takes constant values inside the vortex cores, in particular, $\Phi\left(\vartheta<\vartheta_{c}\right) \approx \Phi_{0}= \pm \pi / 2\left(1-p \vartheta_{c} \ln \vartheta_{c}\right)$. Energy of the vortex state with exception of the core energy $E_{c}$ depends on the core size, $E-E_{c} \propto \vartheta_{c}^{2} u_{c}$. The dependence $\Phi(\vartheta)$ is indicated in Fig. 1 by dashed lines. The approximate solution is in good agreement with the numerical solution of Eq. (3b), where the out-of-surface component was chosen according to Eq. (4). It should be emphasized that the phase of the vortex on a spherical surface gains a coordinate dependence given by Eq. (7) and has the maximum amplitude in the center of each vortex, as opposed to the planar vortex.

For the case of opposite polarities $\left(p_{1}=-p_{2}=p\right)$, the energy reaches its minimum for the trivial solutions (see Appendix B) $\Phi=\pi$ for $p=1$ and $\Phi=0$ for $p=-1$ (as earlier, we consider the case $\vartheta_{c} \ll 1$ ). Such a solution can be considered as a three-dimensional generalization of the well-known onion state in narrow nanorings. The energy of the onion state, $E-E_{c} \propto-\vartheta_{c}$, is lower than that for the vortex state, namely the energy gain is $\Delta E \propto \vartheta_{c}$. Nevertheless, it should be emphasized that these two states are separated by a high energy barrier related to the polarity switching of one of the vortices. Therefore, we suggest that both states can be realized experimentally even at room temperature. To obtain knowingly the onion state, we suggest applying the high-frequency circularly polarized alternating magnetic 

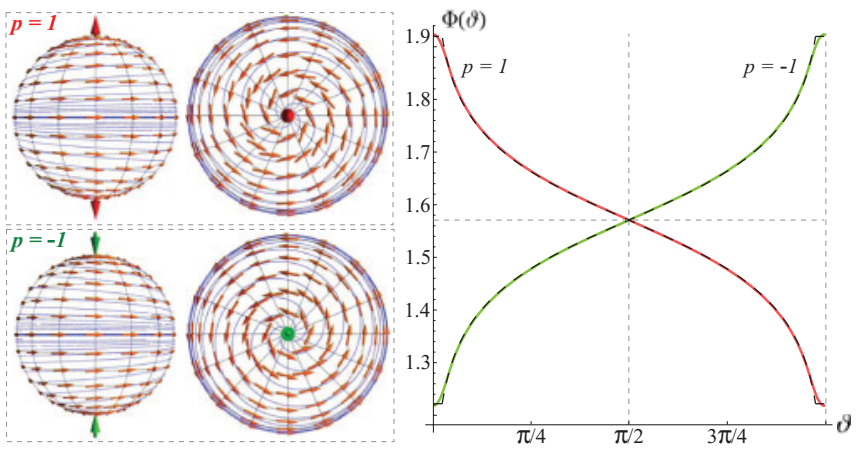

FIG. 1. (Color online) Possible vortex phases $\Phi$ for the case of same polarities $p_{1}=p_{2}=p$ (right inset). Solid lines correspond to the exact numerical solution of Eq. (3b), where the out-of-surface component is chosen in the form of Eq. (4) with $\vartheta_{c}=0.05$. The corresponding approximate solutions (7) are indicated by dashed lines. The corresponding magnetization distribution on the sphere surface is schematically shown in the left inset using arrows and stream lines.

field in the unidirectional switching regime, similarly as was done for disks. ${ }^{26}$ Applying the mentioned magnetic field in a multiple-switching regime, one should expect to obtain the vortex state with a probability slightly less than $1 / 2$.

All possible vortexlike states described above are presented in Fig. 2.

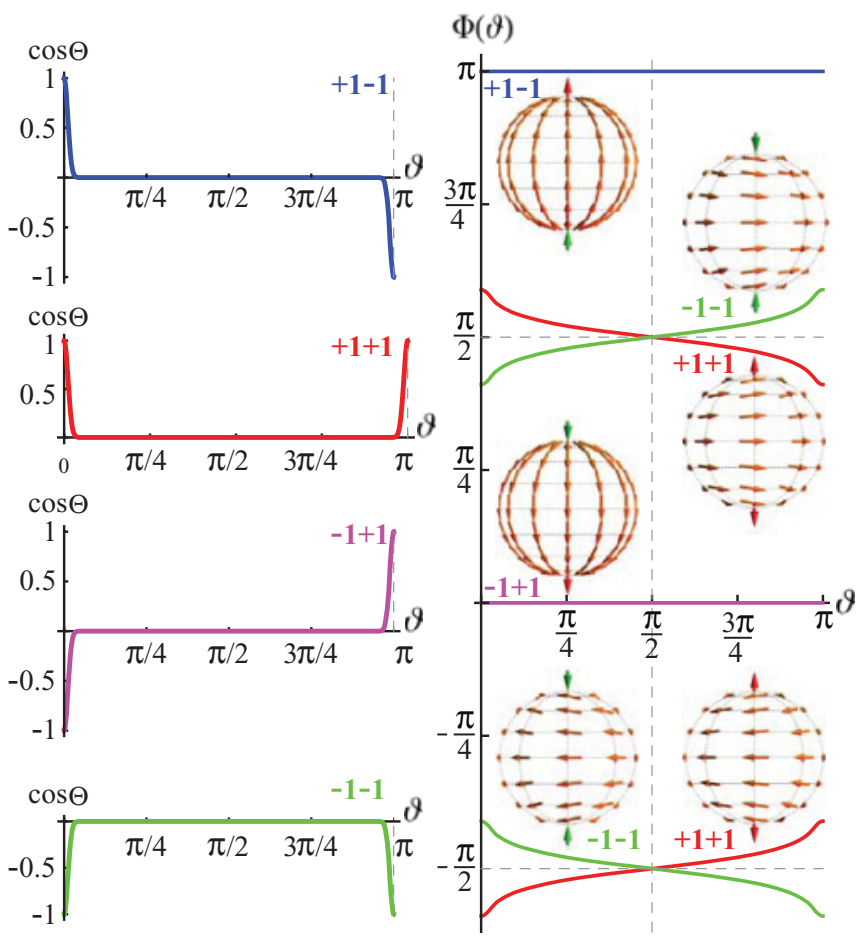

FIG. 2. (Color online) All possible vortex states of the spherical surface. The left column demonstrates the model out-of-surface magnetization distribution given by Eq. (4) for all possible combinations of polarities. The right graph shows the corresponding distributions of the phase $\Phi(\vartheta)$. For the correspondence, we use the notation $p_{1} p_{2}$, e.g., " $+1-1$ " denotes $p_{1}=+1$ and $p_{2}=-1$.

\section{NUMERICAL ANALYSIS}

In this paragraph, a comparison between the obtained analytical results and the exact numerical solutions as well as the micromagnetic simulations is given. The numerical solution of Eq. (3) with the boundary conditions $\Theta(0)=$ $\Theta(\pi)=0$ and $\Phi^{\prime}(0)=\Phi^{\prime}(\pi)=0$ leads to a vortex structure for the case of same polarities, $p_{1}=p_{2}=1$. The obtained out-of-surface structure of the vortex core $\cos \Theta$ is quite close to the model solution (4) when $\vartheta_{c}=\ell / L$ (left graph of Fig. 3). The solution for the vortex phase $\Phi(\vartheta)$ for same boundary conditions corresponds to the twofold degenerated solutions of the type given by Eq. (7) with opposite chiralities. One of such solutions is plotted in the right graph (Fig. 3). The exact solution $\Phi(\vartheta)$ has slightly larger amplitude of turning compared with the model solution. This originates from a larger effective core size $\vartheta_{c}$ of the exact solution compared with the model given in Eq. (4) (left graph of Fig. 3).

In order to verify our results, we performed two types of micromagnetic simulations by using the OOMMF code. ${ }^{27}$ A thin spherical shell was simulated considering (i) local magnetic interaction in the form of Eq. (1) and (ii) exchange and nonlocal magnetostatic interactions. In both cases, the material parameters ${ }^{28}$ were chosen to provide the same ratio of characteristic magnetic length and the sphere radius $\ell / L=$ 0.05. Physically, these two types of simulations are equivalent in case of vanishing thickness, when the magnetostatic interaction can be reduced to the easy-surface anisotropy.

Every simulation was repeated with two initial states: a vortex state with $m_{z}=0$ and an onion state which was slightly deformed to avoid a labile equilibrium. Small areas on the poles gained uniform magnetization along the $z$ axis to control polarities of the vortices. The simulations of type (i) confirm the analytical results with a high accuracy. In the case of same polarities, the system relaxes to the vortex state with the additional turning described by Eq. (7) (line 3 in Fig. 3). For opposite polarities, the system relaxes to the state with $\Phi \equiv \pi$ or $\Phi \equiv 0$, as described above.

According to the simulations of type (ii), in the case where $p_{1}=p_{2}$, the magnetostatic interaction attenuates (but does not suppress) the phenomenon of the vortex phase turning (line 4 in Fig. 3) due to an increased energy of volume magnetostatic charges. In the case of $p_{1}=-p_{2}$, the shell of mentioned size relaxes to the vortex state with $\Phi \approx \pm \pi / 2$ instead of the onion state, which is preferred for spheres of smaller size. ${ }^{29}$ To obtain an approximate criterion of the separation between vortex and onion states, the difference of energies of the onion and vortex states are estimated as follows: $\Delta E=$ $\Delta E_{\mathrm{ms}}+\Delta E_{\mathrm{ex}}$, where $\Delta E_{\mathrm{ms}} \sim L h^{2}$ is the energy increase due to volume magnetostatic charges and $\Delta E_{\mathrm{ex}} \sim-\vartheta_{c} \ell^{2} h$ is the corresponding exchange energy decrease. Thereby, the onion state is energetically preferable when $L^{2} h<\ell^{3}$. The detailed study of ground states of soft-magnetic spherical shells goes beyond the scope of this paper and is the subject of a prospective work.

\section{CONCLUSIONS}

In conclusion, we predict novel features of a magnetic vortex in thin spherical shells in comparison with the well-known 


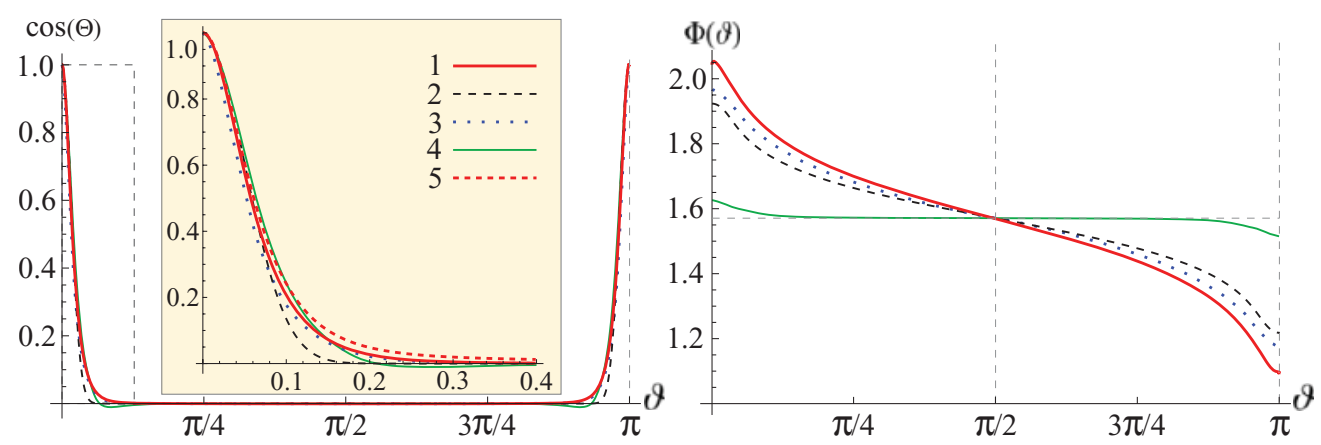

FIG. 3. (Color online) Structure of the vortex state for the case of same polarities $\left(p_{1}=p_{2}=1\right)$ obtained by different methods. Line 1: exact numerical solution of Eq. (3) with boundary conditions $\Theta(0)=\Theta(\pi)=0$ and $\Phi^{\prime}(0)=\Phi^{\prime}(\pi)=0$ and $\ell / L=0.05$. Line 2: (left) the ansatz (4) and (right) corresponding solution of Eq. (3b) where the function $\Theta(\vartheta)$ is determined by Eq. (4) with an angular vortex core size $\vartheta_{c}=\ell / L=0.05$. This line coincides with line " $p=1$ " in Fig. 1. Line 3 and line 4 correspond to micromagnetic simulations of types (i) and (ii), respectively (see text for details). Line 5 in the left graph shows the out-of-surface structure of the vortex core for the case of opposite polarities, when $p_{1}=-p_{2}=1$ and $\Phi \equiv \pi$.

vortex in a planar easy-plane magnet. We show that the vortex on a spherical surface gains a coordinate and polaritydependent turning of its phase. An interplay between topological properties of the vortex, namely its polarity, and the curvature of the underlying surface breaks the degeneration of the phaselike variable $\Phi$ with respect to the rotation by any constant angle $\Phi_{0}$. This degeneration is known as well as for $\pi_{1}$ vortices in different media and for $\pi_{2}$ vortices in flat magnets. It is instructive to note that the angle $\Phi_{0}$ in magnetic nanodisks determines the vortex chirality. ${ }^{30}$ Thus, one can speak about polarity-chirality coupling.

\section{ACKNOWLEDGMENTS}

This work is financed in part via the German Science Foundation (DFG) grant MA 5144/1-1 and DFG Research Unit 1713.

\section{APPENDIX A: ASYMPTOTIC OF FUNCTIONS $\Theta(\vartheta)$ AND $\Phi(\vartheta)$ IN THE NEIGHBORHOOD OF THE VORTEX ORIGIN}

We consider here an asymptotic solution of Eqs. (3) in the neighborhood of point $\vartheta=0$. The functions $\Theta(\vartheta)$ and $\Phi(\vartheta)$ can be presented in form of the Tailor series,

$$
\begin{aligned}
& \Theta \approx \pi h(-p)+p \frac{\vartheta}{\vartheta_{c}}+\sum_{n=2}^{N} a_{n} \vartheta^{n}, \\
& \Phi \approx \Phi_{0}+\sum_{n=1}^{N} b_{n} \vartheta^{n},
\end{aligned}
$$

where $p$ is the vortex polarity and $h(x)$ is the Heaviside function. The expansion (A1a) satisfies the necessary boundary conditions: $\Theta(0)=0$ for $p=+1$ and $\Theta(0)=\pi$ for $p=-1$. To obtain the asymptotic expansion accurate within terms of order $\mathcal{O}\left(\vartheta^{v}\right)$ we can restrict ourselves with $N=v+m$, where $m=2$ is the order of Eqs. (3). We then substitute the series (A1) into (3), expand the obtained equations by the small quantity $\vartheta$, and equate the series coefficients of the same order terms until the order $v$. The obtained system results a relation of expansion coefficients in (A1). For the case $v=2$ the described procedure results in

$$
\begin{aligned}
& a_{2}=b_{1}=0, \\
& b_{2}=-p \frac{\sin \Phi_{0}}{4 \vartheta_{c}} .
\end{aligned}
$$

Thereby, we obtain the following asymptotic expansion accurate within terms of order $\mathcal{O}\left(\vartheta^{2}\right)$ :

$$
\begin{aligned}
& \Theta \approx \pi h(-p)+p \frac{\vartheta}{\vartheta_{c}}, \\
& \Phi \approx \Phi_{0}-p \frac{\sin \Phi_{0}}{4 \vartheta_{c}} \vartheta^{2} .
\end{aligned}
$$

\section{APPENDIX B: VORTEX PHASE SOLUTION $\Phi(\vartheta)$ FOR THE STEPWISE VORTEX SHAPE MODEL}

In the following, we focus on the solution of the Eq. (5). Using the stepwise vortex shape model $m_{r}(u) \approx p_{1}-p_{1} h(u+$ $\left.u_{c}\right)+p_{2} h\left(u-u_{c}\right)$, one can rewrite (5) in the following form:

$$
\begin{aligned}
\partial_{u u} \Phi & =-g(u) \sin \Phi, \\
g(u) & \approx \frac{\pi}{2} \vartheta_{c}\left[p_{1} \delta\left(u+u_{c}\right)-p_{2} \delta\left(u-u_{c}\right)\right],
\end{aligned}
$$

where $\delta(u)$ is the Dirac $\delta$ function. This approximation agrees well with the real peaked form of the $g$ function; see Fig. 4. The general solution of (B1) takes the form,

$$
\begin{aligned}
\Phi(u)= & \Phi_{0}+\Phi_{1} u-\frac{\pi}{2} \vartheta_{c}\left[p_{1} \sin \Phi\left(-u_{c}\right)\left(u+u_{c}\right)_{+}\right. \\
& \left.-p_{2} \sin \Phi\left(u_{c}\right)\left(u-u_{c}\right)_{+}\right]
\end{aligned}
$$

with $u_{+} \equiv u h(u)$ and $h(u)$ being the Heaviside step function. Using the Neumann boundary conditions $\partial_{u} \Phi( \pm \infty)=0$, one can find that $\Phi_{1}=0$ and

$$
p_{1} \sin \Phi\left(-u_{c}\right)=p_{2} \sin \Phi\left(u_{c}\right) .
$$

Substituting (B3) into (B2) results in

$$
\Phi(u)=\Phi_{0}-\frac{\pi}{2} \vartheta_{c} p_{1} \sin \Phi\left(-u_{c}\right)\left[\left(u+u_{c}\right)_{+}-\left(u-u_{c}\right)_{+}\right] .
$$



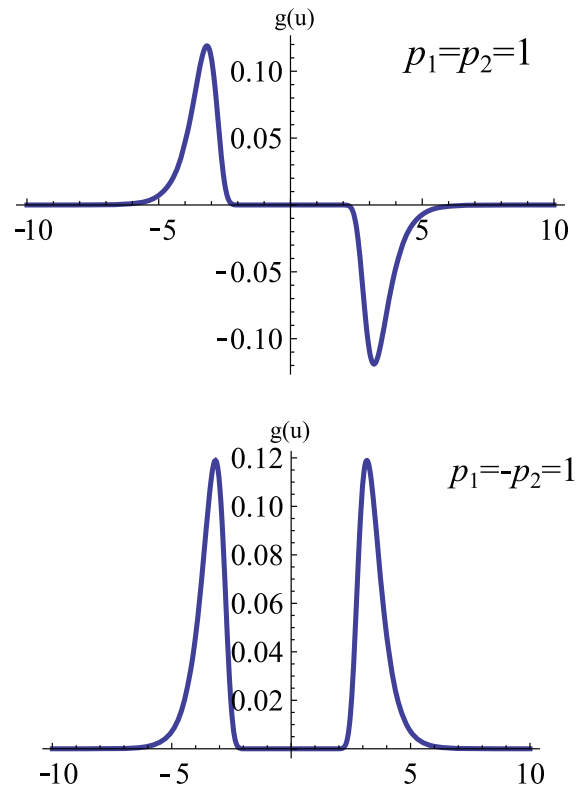

FIG. 4. (Color online) Function $g(u)$ from Eq. (5) for $\vartheta_{c}=0.05$.

Thereby, for the interval $-u_{c}<u<u_{c}$, one can write $\Phi(u)=$ $a u+b$, where constants $a$ and $b$ can be found from the system

$$
\begin{aligned}
p_{1} \sin \left(-a u_{c}+b\right) & =p_{2} \sin \left(a u_{c}+b\right), \\
a & =-\frac{\pi}{2} \vartheta_{c} p_{1} \sin \left(-a u_{c}+b\right) .
\end{aligned}
$$

Further analysis essentially depends on the relative polarities of vortices. For the case of equal polarities $p_{1}=p_{2}=p$ the system (B5) has the following solutions:

$$
\begin{aligned}
& b= \pm \frac{\pi}{2}, \quad a=\mp \frac{\pi}{2} \vartheta_{c} p \cos \left(a u_{c}\right), \\
& a=k \pi, \quad \sin b=(-1)^{k+1} \frac{p k}{2 \vartheta_{c} u_{c}},
\end{aligned}
$$

where $k \in \mathbb{Z}$. Taking into account that $\vartheta_{c} u_{c} \ll 1$, one obtains from (B6b) only the trivial solution $a=0$ and $b=k \pi$. Substituting the obtained solutions into Hamiltonian (2) results in the trivial solution corresponding to the energy maximums and the solutions (B6a) minimizing the energy. Therefore, for the case of equal polarities one obtains the solution (7).

For the case of opposite polarities $p_{1}=-p_{2}=p$ the system (B5) results in

$$
\begin{aligned}
& a=\frac{\pi}{2 u_{c}}(2 k+1), \quad \cos b=(-1)^{k}(2 k+1) \frac{p}{u_{c} v_{c}}, \\
& b=k \pi, \quad \sin \left(a u_{c}\right)=(-1)^{k} a \frac{2 p}{\pi \vartheta_{c}} .
\end{aligned}
$$

Due to the condition $\vartheta_{c} u_{c} \ll 1$ the system (B7) results in the trivial solutions $a=0$ and $b=k \pi$ and, consequently, $\Phi=0$ or $\Phi=\pi$. Analysis of the energy functional shows that for $p=1$ the solution $\Phi=\pi$ and, for $p=-1$, the solution $\Phi=0$ correspond to the energy minimum. *vkravchuk@bitp.kiev.ua

${ }^{1}$ H. Kuratsuji, Phys. Rev. E 85, 031150 (2012).

${ }^{2}$ J. Tempere, V. N. Gladilin, I. F. Silvera, J. T. Devreese, and V. V. Moshchalkov, Phys. Rev. B 79, 134516 (2009).

${ }^{3}$ V. M. Fomin, R. O. Rezaev, and O. G. Schmidt, Nano Lett. 12, 12821287 (2012).

${ }^{4}$ T. Lopez-Leon, V. Koning, K. B. S. Devaiah, V. Vitelli, and A. Fernandez-Nieves, Nat. Phys. 7, 391 (2011).

${ }^{5}$ B. K. Ganser, S. Li, V. Y. Klishko, J. T. Finch, and W. I. Sundquist, Science 283, 80 (1999).

${ }^{6}$ H. T. McMahon and J. L. Gallop, Nature 438, 590 (2005).

${ }^{7}$ M. J. Bowick and L. Giomi, Adv. Phys. 58, 449 (2009).

${ }^{8}$ A. M. Turner, V. Vitelli, and D. R. Nelson, Rev. Mod. Phys. 82, 1301 (2010)

${ }^{9}$ V. Vitelli and A. M. Turner, Phys. Rev. Lett. 93, 215301 (2004).

${ }^{10} \mathrm{G}$. Volovik, The Universe in a Helium Droplet (Oxford University Press, Oxford, 2003).

${ }^{11}$ J.-G. Zhu, Y. Zheng, and G. A. Prinz, J. Appl. Phys. 87, 6668 (2000).

${ }^{12}$ A. P. Guimarães, Principles of Nanomagnetism, NanoScience and Technology (Springer-Verlag, Berlin, 2009).

${ }^{13}$ A. Hubert and R. Schäfer, Magnetic Domains: The Analysis of Magnetic Microstructures (Springer-Verlag, Berlin, 1998).

${ }^{14}$ K. Nakano, D. Chiba, N. Ohshima, S. Kasai, T. Sato, Y. Nakatani, K. Sekiguchi, K. Kobayashi, and T. Ono, Appl. Phys. Lett. 99, 262505 (2011); X. Z. Yu, N. Kanazawa, Y. Onose, K. Kimoto, W. Z. Zhang, S. Ishiwata, Y. Matsui, and Y. Tokura, Nat. Mater. 10, 106 (2011).

${ }^{15}$ M. Curcic, B. Van Waeyenberge, A. Vansteenkiste, M. Weigand, V. Sackmann, H. Stoll, M. Fähnle, T. Tyliszczak, G. Woltersdorf,
C. H. Back, and G. Schütz, Phys. Rev. Lett. 101, 197204 (2008).

${ }^{16}$ K. W. Chou, A. Puzic, H. Stoll, D. Dolgos, G. Schutz, B. V. Waeyenberge, A. Vansteenkiste, T. Tyliszczak, G. Woltersdorf, and C. H. Back, Appl. Phys. Lett. 90, 202505 (2007).

${ }^{17}$ A. Vansteenkiste, M. Weigand, M. Curcic, H. Stoll, G. Schtz, and B. V. Waeyenberge, New J. Phys. 11, 063006 (2009).

${ }^{18}$ A. Saxena, R. Dandoloff, and T. Lookman, Physica A 261, 13 (1998); P. Landeros, O. J. Suarez, A. Cuchillo, and P. Vargas, Phys. Rev. B 79, 024404 (2009); R. Dandoloff, S. Villain-Guillot, A. Saxena, and A. R. Bishop, Phys. Rev. Lett. 74, 813 (1995);

S. Villain-Guillot, R. Dandoloff, A. Saxena, and A. R. Bishop, Phys. Rev. B 52, 6712 (1995).

${ }^{19}$ V. L. Carvalho-Santos, A. R. Moura, W. A. Moura-Melo, and A. R. Pereira, Phys. Rev. B 77, 134450 (2008); V. L. CarvalhoSantos, W. A. Moura-Melo, and A. R. Pereira, J. Appl. Phys. 108, 094310 (2010)

${ }^{20}$ W. A. Freitas, W. A. Moura-Melo, and A. Pereira, Phys. Lett. A 336, 412 (2005); W. Moura-Melo, A. Pereira, L. Mól, and A. Pires, ibid. 360, 472 (2007).

${ }^{21}$ M. LoBue, F. Mazaleyrat, M. Ammar, R. Barrué, Y. Champion, S. Faure, M. Hÿtch, E. Snoeck, J. Steiner, and F. Alouges, J. Magn. Magn. Mater. 322, 1290 (2010); P. Barpanda, T. Kasama, R. E. Dunin-Borkowski, M. R. Scheinfein, and A. S. Arrott, J. Appl. Phys. 99, 08G103 (2006).

${ }^{22}$ M. Albrecht, G. Hu, I. L. Guhr, T. C. Ulbrich, J. Boneberg, P. Leiderer, and G. Schatz, Nat. Mater. 4, 203 (2005); T. C. Ulbrich, D. Makarov, G. Hu, I. L. Guhr, D. Suess, T. Schrefl, and M. Albrecht, Phys. Rev. Lett. 96, 077202 (2006); M. V. Sapozhnikov, 
O. L. Ermolaeva, B. G. Gribkov, I. M. Nefedov, I. R. Karetnikova, S. A. Gusev, V. V. Rogov, B. B. Troitskii, and L. V. Khokhlova, Phys. Rev. B 85, 054402 (2012).

${ }^{23}$ R. Dandoloff and A. Saxena, J. Phys. A: Math. Theor. 44, 045203 (2011).

${ }^{24} \mathrm{We}$ focus here only on the vortex solutions and do not consider the other possible solutions which can appear in case $\ell \approx L$.

${ }^{25}$ E. Feldtkeller and H. Thomas, Z. Phys. B: Condens. Matter 4, 8 (1965).

${ }^{26}$ V. P. Kravchuk, D. D. Sheka, Y. Gaididei, and F. G. Mertens, J. Appl. Phys. 102, 043908 (2007).

27 “The Object Oriented MicroMagnetic Framework", developed by M. J. Donahue and D. Porter mainly, from NIST. We used the 3D version of the $1.2 \alpha 4$ release.
28 The spherical shell with an inner radius $L=150 \mathrm{~nm}$ and a thickness $h=15 \mathrm{~nm}$ was simulated using a unit cell size $\Delta=5$ $\mathrm{nm}$. For both types of simulations, the exchange constant $A=$ $1.3 \times 10^{-11} \mathrm{~J} / \mathrm{m}$ and the saturation magnetization $M_{S}=6 \times 10^{5}$ $\mathrm{A} / \mathrm{m}$ were used. In simulations of type (i) we used an uniaxial anisotropy with a spatially varying hard axis orientated along the radial vector $\boldsymbol{e}_{r}$ and an anisotropy constant $K=-2.2 \times$ $10^{5} \mathrm{~J} / \mathrm{m}^{3}$. For both types of simulations the characteristic magnetic length were approximately equal: $\sqrt{A /|K|} \approx \sqrt{A / 2 \pi M_{S}^{2}} \approx$ $7.6 \mathrm{~nm}$.

${ }^{29}$ For example, a spherical shell with an inner radius $L=20 \mathrm{~nm}$ and a thickness $h=4 \mathrm{~nm}$.

${ }^{30}$ Y. Gaididei, D. D. Sheka, and F. G. Mertens, Appl. Phys. Lett. 92, 012503 (2008). 